\title{
Re: Causes of Hospital Readmissions after Urologic Cancer Surgery
}

\section{Schmid M1, Chiang HA2, Sood A3, Campbell L3, Chun FK4, Dalela D3, Okwara J2, Sammon JD3, Kibel AS2, Menon M3, Fisch M4, Trinh QD2}

${ }^{1}$ Center for Surgery and Public Health; Clinic of Urologic Surgery and; Brigham and Women's Hospital, Harvard Medical School, Boston, Massachusetts and Department of Urology, University Medical Center Hamburg-Eppendorf, Hamburg, Germany

2Brigham and Women's Hospital, Harvard Medical School, Center for Surgery and Public Health and Clinic of Urologic Surgery, Boston, Massachusetts

3Vattikuti Urology Institute, Henry Ford Health System, Detroit, Michigan

4 University Medical Center Hamburg-Eppendorf, Clinic of Urology, Hamburg, Germany

Urol Oncol 2016;34:236.e1-236.e11. doi: 10.1016/j.urolonc.2015.11.019. Epub 2015 Dec 23.

\section{EDITORIAL COMMENT}

Unplanned readmissions after surgery are a cause for anxiety and inconvenience for the patient and financial burden for the healthcare system. It has been advocated that unplanned readmission rates can be used as a metric for quality of patient care. In the light of the changes in health care policy with an emphasis on reducing readmissions, Schmid and coworkers used the American College of Surgeons National Surgical Quality Improvement Program database to examine the causes and predictors of readmissions after urologic oncology surgical procedures, namely radical prostatectomy (RP), radical nephrectomy (RN), partial nephrectomy (PN), and radical cystectomy (RC). The overall unplanned 30-day readmission rate was 5.5\%. Approximately two thirds of readmissions occurred within the first 10 days following hospital discharge. Comparing with the other procedures, RC patients experienced the highest rate (15.9\%) for readmissions. For $\mathrm{RP}$ and PN, minimally invasive approach was associated with decreased odds for readmissions. Better analyzing the causes and developing strategies to prevent early readmissions after urologic cancer surgery would undoubtedly help to improve patient outcomes and decrease the health care costs.

Özgür Yaycıoğlu, MD

\section{Urooncology}

doi: $10.4274 /$ jus.2016.03.022

\section{Re: Metastatic Prostate Cancer in Men Initially Treated with Active Surveillance Yamamoto T, Musunuru B, Vesprini D, Zhang L, Ghanem G, Loblaw A, Klotz L \\ Sunnybrook Health Sciences Centre, University of Toronto, Department of Urology, Ontario, Canada; and Sunnybrook Health Sciences Centre, Clinic of Radiation Oncolog, Ontario, Canada}

J Urol 2016;195:1409-1414. doi: 10.1016/j.juro.2015.11.075. Epub 2015 Dec 18.

\section{EDITORIAL COMMENT}

Active surveillance (AS) in prostate cancer has been advocated as a treatment modality to reduce overtreatment of patients with clinically insignificant disease while appropriately offering radical treatment to those in whom the disease is reclassified as high-risk during surveillance. Yamamoto and coworkers have analyzed their prospective cohort of 993 patients treated with AS and analyzed the characteristics of those who eventually progressed to metastatic disease. Out of 980 evaluable patients, 133 (13.6\%) had Gleason score (GS) 7 disease. During AS, 30 patients (3.1\%) developed metastases. Of note, metastases developed in 13 of $133(10 \%)$ patients with GS 7 disease. The median time to metastasis was 6.3 years. On univariate analysis, GS 7, number of positive cores, core positivity greater than 50\% at initial biopsy, intermediate risk group, and short prostate-specific antigen (PSA) doubling time (DT) were significant risk factors for metastases. On multivariate analysis, GS 7, a total of 3 or more positive cores, and PSA DT remained significant. The presence of Gleason pattern 4 on diagnostic biopsy conferred a threefold to fourfold increased risk of metastatic disease. Even though the authors concluded that GS seven patients should be offered AS with caution, the safety of such a suggestion is quite questionable. 